\title{
Integrating Factors and First Integrals for Liénard Type and Frequency-Damped Oscillators
}

\author{
Emrullah Yaşar \\ Department of Mathematics, Faculty of Arts and Sciences, Uludag University, 16059 Bursa, Turkey \\ Correspondence should be addressed to Emrullah Yaşar, eyasar@uludag.edu.tr
}

Received 25 January 2011; Revised 11 April 2011; Accepted 12 April 2011

Academic Editor: Alexei Mailybaev

Copyright (C) 2011 Emrullah Yaşar. This is an open access article distributed under the Creative Commons Attribution License, which permits unrestricted use, distribution, and reproduction in any medium, provided the original work is properly cited.

\begin{abstract}
We consider Liénard type and frequency-damped oscillator equations. Integrating factors and the associated first integrals are derived from the method to compute $\lambda$-symmetries and the associated reduction algorithm. The knowledge of a $\lambda$-symmetry of the equation permits the determination of an integrating factor or a first integral by means of coupled first-order linear systems of partial differential equations. We will compare our results with those gained by the other methods.
\end{abstract}

\section{Introduction}

Integrating factors and first integrals are powerful tools in the study of ordinary differential equations (ODEs). In this area, we observe plenty of studies. Some of them are listed in [1-8]. Several authors have obtained necessary and sufficient conditions for a function $\mu(t, x, \dot{x})$ to be an integrating factor of a second-order ODEs. Most of their approaches rest on the fact that the multiplication of function $\mu$ with second-order ODE is a total derivative, and therefore its variational derivative is null. As a consequence, integrating factors can be determined as solutions of a second-order linear system of partial differential equations (PDEs). Since solving this system is usually a more difficult task than solving the original ODE, many studies have been done to investigate special classes of integrating factors, through specific ansatze for $\mu$ [9]. For instance, in [10] Anco and Bluman derive integrating factors as the solutions of the adjoint equation of the linearized equation and an additional equation that describes an extra adjoint-invariance condition.

In their recent papers, Muriel and Romero [11, 12] presented what they termed a systematic algorithm for the construction of integrating factors of the form $\mu(t, x, \dot{x})$ for second-order ODEs. With this new powerful method, integrating factors and the associated first integrals are derived from the method to compute $\lambda$-symmetries and the associated 
reduction algorithm. In addition, the knowledge of a $\lambda$-symmetry of the equation permits the determination of an integrating factor or a first integral by means of coupled first-order linear systems of PDEs.

The main purpose of this paper is to study integrating factors and first integrals of two important oscillation equations which are Liénard type and frequency-damped oscillation equations by the $\lambda$-symmetry methods. An interesting feature of our study is that the method of [10] does not produce integrating factors for Liénard type oscillation equation; however, $\lambda$-symmetry methods yield integrating factor and first integral. Moreover, we obtain new integrating factor by $\lambda$-symmetry methods different from that which is previously derived by [10] for frequency-damped oscillation equation.

The paper is structured thusly. In Section 2, we present the necessary preliminaries. This section is devoted to integrating factors and the associated first integrals which are derived from the method to compute $\lambda$-symmetries and the associated reduction algorithm. In addition, we introduce Anco and Bluman's method [10].

In Section 3, we study the $\lambda$-symmetries, integrating factors, first integrals for Liénard type, and frequency-damped oscillator equations. In addition, we make comparisons between two methods which are pointed out briefly above. The final section includes some conclusions.

\section{Necessary Preliminaries}

We first present notation to be used and recall the definitions and theorems that appear in $[10,11]$.

Consider an $n$ th-order ordinary differential equation

$$
\tilde{\Delta}\left(t, x^{(n)}\right)=0,
$$

where variables $(t, x)$ are in some open set $M \subset T \times X \simeq \mathbb{R}^{2}$. For $k \in \mathbb{N}, M^{k} \subset T \times X^{(k)}$ denotes the corresponding $k$-jet space, and the elements of $M^{(k)}$ are denoted by $\left(t, x^{(k)}\right)=$ $(t, x, \dot{x}, \ldots, \stackrel{k}{x})$.

Suppose that (2.1) admits an integrating factor, $\mu\left(t, x^{(k)}\right)$, for some $k$ such that $0 \leq k \leq$ $n-1$. The multiplication by $\mu$ converts the left-hand side of (2.1) into the total derivative of some function $\Delta\left(t, x^{(n-1)}\right)$,

$$
\mu\left(t, x^{(k)}\right) \cdot \tilde{\Delta}\left(t, x^{(n)}\right)=D_{t}\left(\Delta\left(t, x^{(n-1)}\right)\right) .
$$

The exact equation $D_{t}\left(\Delta\left(t, x^{(n-1)}\right)\right)=0$ admits a $\lambda$-symmetry, and the trivial reduction of order $\Delta\left(t, x^{(n-1)}\right)=C, C \in \mathbb{R}$, appears as a consequence of the reduction algorithm associated to that $\lambda$-symmetry [13]. If $\lambda \in C^{\infty}\left(M^{(k)}\right), 0 \leq k \leq n-1$, is any solution of the partial differential equation

$$
\sum_{i=0}^{n-1}\left(D_{t}+\lambda\right)^{i}(1) \frac{\partial \Delta}{\partial x^{(i)}}=0 \quad \text { when } x^{(n)}=F\left(t, x^{(n-1)}\right),
$$


then the vector field $v=\partial_{x}$ is a $\lambda$-symmetry of (2.1) where $F$ is an analytic function of its arguments in some open subset $M^{(n-1)}$, and it appears when (2.1) is locally written in explicit form.

Theorem 2.1 (see [12]). Assume that (2.1) is an nth-order ordinary differential equation that admits an integrating factor $\mu$ such that $\left.\mu\right|_{\tilde{\Delta}=0} \neq 0$. If $\lambda$ is any particular solution of (2.3), then the vector field $v=\partial_{x}$ is a $\lambda$-symmetry of (2.1).

An algorithm to obtain an integrating factor (or first integral) of a given ODE once a $\lambda$-symmetry is known has been derived in [12]. For second-order equations, the method reads as follows.

For $n=2$, the corresponding second-order ODEs can be written in explicit form as

$$
\ddot{x}=F(t, x, \dot{x}) .
$$

We denote by $A=\partial_{t}+\dot{x} \partial_{x}+F(t, x, \dot{x}) \partial_{\dot{x}}$ the vector field associated with (2.4). In terms of $A$, a first integral of (2.4) is any function $I(t, x, \dot{x})$ such that $A(I)=0$.

Let $v$ be a $\lambda$-symmetry of $(2.4)$, Then $w(t, x, \dot{x})$ is a first-order invariant of $v^{[\lambda,(1)]}$, that is, any particular solution of the equation

$$
w_{x}+w_{\dot{x}} \lambda(t, x, \dot{x})=0 .
$$

The reduction process associated to the $\lambda$-symmetry $v$ gives a first-order reduced equation of the form $\Delta_{R}(t, x, \dot{x})=0$, the general solution of which is implicitly given by an equation of the form $G(t, w)=C, C \in \mathbb{R}$. It is clear that $D_{t}(G(t, w(t, x, \dot{x}))=0$ is an equivalent form of (2.4). Therefore,

$$
\mu(t, x, \dot{x})=G_{w}(t, x, w(t, x, \dot{x})) \cdot w_{\dot{x}}(t, x, \dot{x})
$$

is an integrating factor of (2.4).

For second-order ODEs, there is a correspondence between $\lambda$-symmetries and first integrals in the sense of Theorem 2 in [11].

Theorem 2.2 (see [11]). (a) If $I(t, x, \dot{x})$ is a first integral of (2.4), then the vector field $v=\partial_{x}$ is a $\lambda$-symmetry of (2.4) for $\lambda=-I_{x} / I_{\dot{x}}$ and $v^{[\lambda,(1)]} I=0$.

(b) Conversely, if $v=\partial_{x}$ is a $\lambda$-symmetry of (2.4) for some function $\lambda(t, x, \dot{x})$, then there exists a first integral $I(t, x, \dot{x})$ of $(2.4)$ such that $v^{[\lambda,(1)]} I=0$.

Theorem 2.2 works when $v=\partial_{x}$ is a $\lambda$-symmetry. If $I$ is a first integral of (2.4), then $\mu=I_{\dot{x}}$ is an integrating factor of (2.4) and $-\mu \phi=I_{x}+\dot{x} I_{x}$. If $I$ is also a first integral of $v^{[\lambda,(1)]}$ for some function $\lambda(t, x, \dot{x})$, then $I_{x}=-\lambda I_{\dot{x}}=-\lambda \mu$ and the system

$$
I_{x}=\mu(\lambda \dot{x}-\phi), \quad I_{x}=-\lambda \mu, \quad I_{\dot{x}}=\mu
$$

is compatible; that is, when $\lambda(t, x, \dot{x})$ is such that $v=\partial_{x}$ is $\lambda$-symmetry, we know that system (2.7) is compatible. Therefore, system (2.7) could be used to obtain through a line integral a first integral of (2.4) associated with $\mu$. 
Similarly, for second-order ODEs, there is a correspondence between integrating factors and Lie point symmetries in the sense of Theorem 7 in [11].

Theorem 2.3 (see [11]). If $v$ is a Lie point symmetry of (2.4) and $Q=\eta-\xi \dot{x}$ is its characteristic, then $\bar{v}=\partial_{x}$ is a $\lambda$-symmetry of (2.4) for $\lambda=A(Q) / Q$, and any solution of the first-order linear system

$$
A(\mu)+\left(F_{\dot{x}}-\frac{A(Q)}{Q}\right) \mu=0, \quad \mu_{x}+\left(\frac{A(Q)}{Q} \mu\right)_{\dot{x}}=0
$$

is an integrating factor of (2.4).

Now, we briefly summarize the approach which is presented in [10].

The linearized ODE for (2.4) is given by

$$
L[x] \sigma=\frac{d^{2} \sigma}{d t^{2}}-F_{\dot{x}} \frac{d \sigma}{d t}-F_{x} \sigma=0,
$$

and the corresponding adjoint ODE is

$$
L^{*}[x] \varpi=\frac{d^{2} \varpi}{d t^{2}}+\frac{d}{d t}\left(F_{\dot{x}} \varpi\right)-F_{x} \varpi=0 .
$$

The solutions $\varpi=\mu(t, x, \dot{x})$ of ODE (2.10), holding for any $x(t)$ satisfying the second-order ODE (2.4), are the adjoint symmetries of (2.4). The adjoint invariance conditions for $\mu(t, x, \dot{x})$ to be an integrating factor of (2.4) are

$$
\begin{aligned}
& \mu_{t t}+ 2 \dot{x} \mu_{t x}+2 F \mu_{t \dot{x}}+(\dot{x})^{2} \mu_{x x}+2 \dot{x} F \mu_{x \dot{x}}+F^{2} \mu_{\dot{x} \dot{x}}+\left(F_{t}+\dot{x} F_{x}+2 F F_{\dot{x}}\right) \mu_{\dot{x}} \\
&+\left(F+\dot{x} F_{\dot{x}}\right) \mu_{x}+F_{\dot{x}} \mu_{t}+\left(F_{t \dot{x}}+\dot{x} F_{\dot{x} \dot{x}}+F F_{\dot{x} \dot{x}}-F_{x}\right) \mu=0, \\
& \mu_{t \dot{x}}+\dot{x} \mu_{x \dot{x}}+F \mu_{\dot{x} \dot{x}}+2 F_{\dot{x}} \mu_{\dot{x}}+2 \mu_{x}+F_{\dot{x} \dot{x}} \mu=0 .
\end{aligned}
$$

Equations (2.11)-(2.12) must hold for arbitrary values of $t, x, \dot{x}$.

\section{3. $\lambda$-Symmetries, Integrating Factors, and First Integrals}

\subsection{Liénard Type Oscillator Equation}

Liénard type nonlinear oscillators of the form

$$
\ddot{x}+k x \dot{x}+\frac{k^{2}}{9} x^{3}+a x=0
$$

and their generalizations are widely used in applications in the context of nonlinear oscillations [14-18]. 
We know that (3.1), that is,

$$
\ddot{x}=-k x \dot{x}-\frac{k^{2}}{9} x^{3}-a x=F,
$$

admits the vector field $v=\partial / \partial x$ as a $\lambda$-symmetry, where $\lambda$ is any particular solution to the following equation:

$$
\left(D_{t}+\lambda\right)^{2}(1)=\sum_{i=0}^{1}\left(D_{t}+\lambda\right)^{i}(1) \frac{\partial F}{\partial x^{(i)}} \quad \text { when } \ddot{x}=-k x \dot{x}-\frac{k^{2}}{9} x^{3}-a x=F,
$$

or equivalently, $D_{t} \lambda+\lambda^{2}=\lambda_{t}+\dot{x} \lambda_{x}+\left(-k x \dot{x}-\left(k^{2} / 9\right) x^{3}-a x\right) \lambda_{\dot{x}}+\lambda^{2}=-k \dot{x}-\left(k^{2} / 3\right) x^{2}-a-k \lambda x$. Therefore, we obtain the following equation:

$$
\lambda_{t}+\dot{x} \lambda_{x}+\left(-k x \dot{x}-\frac{k^{2}}{9} x^{3}-a x\right) \lambda_{\dot{x}}+\lambda^{2}+k \lambda x+k \dot{x}+\frac{k^{2}}{3} x^{2}+a=0,
$$

that corresponds to (3.3).

For the sake of simplicity, we try to find a solution $\lambda$ of (3.4) of the form $\lambda(t, x, \dot{x})=$ $\lambda_{1}(t, x) \dot{x}+\lambda_{2}(t, x)$. This ansatz leads to the following system:

$$
\begin{gathered}
\lambda_{1_{t}}+\lambda_{2_{x}}+2 \lambda_{1} \lambda_{2}+k=0 \\
\lambda_{1_{x}}+\lambda_{1}^{2}=0 \\
\lambda_{2_{t}}-\frac{k^{2}}{9} \lambda_{1} x^{3}-a \lambda_{1} x+\lambda_{2}^{2}+k \lambda_{2} x+\frac{k^{2}}{3} x^{2}+a=0 .
\end{gathered}
$$

A particular solution of the second equation is given by $\lambda_{1}=1 / x$. The first and third equations become

$$
\begin{gathered}
\lambda_{2_{x}}+\frac{2}{x} \lambda_{2}+k=0 \\
\lambda_{2_{t}}+\frac{2 k^{2}}{9} x^{2}+\lambda_{2}^{2}+k \lambda_{2} x=0 .
\end{gathered}
$$

The general solution of the first equation is given by $\lambda_{2}(t, x)=-k x / 3+x^{-2} \lambda_{21}(t)$. Since the last equation becomes $x^{-1} \lambda_{21}^{\prime}(t)+x^{-3} \lambda_{21}(t)^{2}+(1 / 3) k \lambda_{21}(t)=0$, we can choose $\lambda_{21}(t)=0$. In consequence, $v=\partial / \partial x$ is a $\lambda$-symmetry for $\lambda(t, x, \dot{x})=(1 / x) \dot{x}-k x / 3$.

In order to find an integrating factor associated to $\lambda$, we must find a first-order invariant $w(t, x, \dot{x})$ of $v^{[\lambda,(1)]}$. The equation that corresponds to $(2.5)$ is

$$
w_{x}+\left(\frac{1}{x} \dot{x}-\frac{k x}{3}\right) w_{\dot{x}}=0
$$


It is clear that $w(t, x, \dot{x})=-(1 / x) \dot{x}-k x / 3$ is a solution of (3.7). In terms of $\left\{t, w, w_{t}\right\},(3.1)$ becomes $w_{t}-w^{2}-a=0$, the general solution of which is given by $(1 / \sqrt{a}) \operatorname{Arctan}(w / \sqrt{a})=$ $t+c, c \in \mathbb{R}$. According to (2.6), an integrating factor is given by

$$
\mu(t, x, \dot{x})=G_{w}(t, x, w(t, x, \dot{x})) \cdot w_{\dot{x}}=-\frac{1}{\left(a+(\dot{x} / x+k x / 3)^{2}\right) x}
$$

We observe that the method we have followed not only provides the integrating factor but also gives the conserved form of the equation without additional computation. The conserved form of the resulting equation is given by

$$
D_{t}\left(\frac{1}{\sqrt{a}} \operatorname{Arctan}\left(-\frac{1}{\sqrt{a} x} \dot{x}-\frac{k x}{3 \sqrt{a}}\right)\right)=0
$$

We note that (3.1) is a particular case of a family of equations

$$
\ddot{x}+a_{1}(x) \dot{x}+a_{0}(x)=0,
$$

with $a_{1}(x)=k x, a_{0}(x)=\left(k^{2} / 9\right) x^{3}+a x$ appearing in [19]. Muriel and Romero in that reference suggest a well-defined algorithm through general Sundman transformations for the class of nonlinear second-order ODEs. They propose considering the problem of linearization through nonlocal transformations from the point of the $\lambda$-symmetries admitted by the equation and their associated first integrals. In our study without considering nonlocal transformations, integrating factors and the associated first integrals are derived from the method to compute $\lambda$-symmetries and the associated reduction algorithm. Moreover, our results (3.8) and (3.9) are compatible with the results obtained in [19].

Now, we try to obtain the integrating factor of (3.1) with the method of [10]. The ODE (3.1) is not self-adjoint, so that its adjoint symmetries are not symmetries.

Here, the adjoint symmetry determining equation (2.11) for $\varpi=\mu(t, x, \dot{x})$ is the following;

$$
\begin{aligned}
\mu_{t t}+ & 2 \dot{x} \mu_{t x}+2\left(-k x \dot{x}-\frac{k^{2}}{9} x^{3}-a x\right) \mu_{t \dot{x}}+\dot{x}^{2} \mu_{x x}+2 \dot{x}\left(-k x \dot{x}-\frac{k^{2}}{9} x^{3}-a x\right) \mu_{x \dot{x}} \\
& \left.+\left(-k x \dot{x}-\frac{k^{2}}{9} x^{3}-a x\right)^{2} \mu_{\dot{x} \dot{x}}+\dot{x}\left(-k \dot{x}-\frac{1}{3} k^{2} x^{2}-a\right)+2\left(-k x \dot{x}-\frac{k^{2}}{9} x^{3}-a x\right)(-k x)\right] \mu_{\dot{x}} \\
& +\left[\left(-k x \dot{x}-\frac{k^{2}}{9} x^{3}-a x\right)+\dot{x}(-k x)\right] \mu_{x}+(-k x) \mu_{t}+\left[-k \dot{x}-\left(-k \dot{x}-\frac{1}{3} k^{2} x^{2}-a\right)\right] \mu=0 .
\end{aligned}
$$

The extra adjoint-invariance determining equation (2.12) becomes

$$
\mu_{t \dot{x}}+\dot{x} \mu_{x \dot{x}}+\left(-k x \dot{x}-\frac{k^{2}}{9} x^{3}-a x\right) \mu_{\dot{x} \dot{x}}+2(-k x) \mu_{\dot{x}}+2 \mu_{x}=0 .
$$


If we consider the special case $\mu=\mu(t, x)$, then (3.11) becomes

$$
\dot{x}^{2} \mu_{x x}+\dot{x}\left[2 \mu_{t x}-k x \mu_{x}-k x \mu_{x}\right]+\left[\mu_{t t}-\left(\frac{k^{2}}{9} x^{3}+a x\right) \mu_{x}-k x \mu_{t}+\left(\frac{1}{3} k^{2} x^{2}+a\right) \mu\right]=0 .
$$

Seperating by monomials, that is,

$$
\begin{gathered}
\dot{x}^{2}: \mu_{x x}=0, \\
\dot{x}: 2 \mu_{t x}-2 x k \mu_{x}=0 \\
1: \mu_{t t}-\left(\frac{k^{2}}{9} x^{3}+a x\right) \mu_{x}-k x \mu_{t}+\left(\frac{1}{3} k^{2} x^{2}+a\right) \mu=0
\end{gathered}
$$

we get $\mu=0$. The situations for $\mu=\mu(x, \dot{x})$ and $\mu=\mu(t, \dot{x})$ are a more difficult task than solving the original ODE.

\subsection{Frequency-Damped Oscillator Equation}

We consider the frequency-damped oscillator equation

$$
\ddot{x}+x \dot{x}^{2}=0
$$

studied by Gordon [20], Sarlet et al. [21], and Mimura and Nono [22].

It is clear that $v=\partial / \partial t$ is a Lie point symmetry of (3.15). The vector field associated with (3.15) is

$$
A=\frac{\partial}{\partial t}+\dot{x} \frac{\partial}{\partial x}-\left(x \dot{x}^{2}\right) \frac{\partial}{\partial \dot{x}}
$$

and the characteristic of $v$ is $Q=-\dot{x}$. The corresponding second equation of (2.8) becomes

$$
\mu_{x}-x \dot{x} \mu_{\dot{x}}-x \mu=0
$$

The solution of (3.17) can be obtained by the characteristic method of Lagrange

$$
\mu(t, x, \dot{x})=e^{x^{2} / 2} M\left(t, \dot{x} e^{x^{2} / 2}\right)
$$

where $M$ is an arbitrary function of $t$ and $w=\dot{x} e^{x^{2} / 2}$. Since $\mu$ must also satisfy the first equation in (2.8),

$$
M_{t}(t, w)=0 .
$$


This implies that $M$ depends only on $w$ and

$$
\mu(t, x, \dot{x})=e^{x^{2} / 2} M\left(\dot{x} e^{x^{2} / 2}\right)
$$

is an integrating factor of (3.15), where $M$ is an arbitrary function.

In order to find a first integral $I$ of (3.15) such that $I_{\dot{x}}=\mu$, we must solve the system that corresponds to (2.7). If, for example, we choose $M(w)=w$, then we get the particular integrating factor

$$
\mu(t, x, \dot{x})=\dot{x} e^{x^{2}}
$$

The above integrating factor is different from the previously derived integrating factors for (3.15) by Anco and Bluman [10].

Integrating factor (3.21) of (3.15), the corresponding system, (2.7), becomes

$$
I_{t}=0, \quad I_{x}=x \dot{x}^{2} e^{x^{2}}, \quad I_{\dot{x}}=\dot{x} e^{x^{2}} .
$$

By evaluating the corresponding line integral, we get the general solution of (3.22) and a class of first integrals of (3.15),

$$
I(t, x, \dot{x})=\frac{\dot{x}^{2}}{2} e^{x^{2}}+C, \quad(C \in \mathbb{R})
$$

We note that (3.15) is a particular case of a family of equations

$$
\ddot{x}+a_{2}(x) \dot{x}^{2}+a_{1}(t) \dot{x}=0,
$$

with $a_{2}(x)=x, a_{1}(t)=0$ appearing in [23]. The authors characterized the (3.24) that admits first integrals of the form $A(t, x) \dot{x}+B(t, x)$ through an easy-to-check criterion expressed in terms of functions $S_{1}, S_{2}, S_{3}$, and $S_{4}$ given by (3.3) and (3.4)-(3.6) in [23]. We could obtained our results (3.21) and (3.23) using this novel approach. Moreover, (3.15) was studied by the same authors from the point of view of linearizations through local and nonlocal transformations in [24]. Our results (3.21) and (3.23) are compatible with the results obtained in [24].

\section{Conclusions}

In this paper, we derived integrating factors and first integrals for Lienard type and frequency-damped oscillator equations by $\lambda$-symmetry approach. From the $\lambda$-symmetry and the associated algorithm of reduction, the integrating factor (3.8) and the associated first integral (3.9) were derived for Liénard type equation. We also show the difficulties to get integrating factors for Liénard type equation by the other methods.

The knowledge of a $\lambda$-symmetry of the equation permits the determination of an integrating factor or a first integral by means of coupled first-order linear systems (2.8) of 
partial differential equations. Solving this system, we obtain the integrating factor (3.21) and the associated first integral (3.23) for frequency-damped oscillator equation (3.15).

\section{Acknowledgment}

The author would like to thank the reviewers for their comments that helped improve the manuscript.

\section{References}

[1] N. H. Ibragimov, A Practical Course in Differential Equations and Mathematical Modelling, ALGA Publications, Karlskrona, Sweden, 2006.

[2] G. W. Bluman and S. C. Anco, Symmetry and Integration Methods for Differential Equations, vol. 154 of Applied Mathematical Sciences, Springer, New York, NY, USA, 2002.

[3] N. H. Ibragimov, "Integrating factors, adjoint equations and Lagrangians," Journal of Mathematical Analysis and Applications, vol. 318, no. 2, pp. 742-757, 2006.

[4] N. H. Ibragimov, "Classical and new results on integrating factors," Archives of ALGA, vol. 5, pp. 121-124, 2008.

[5] K. Gehrs, "Integrating factors of some classes of third-order ODEs," Applied Mathematics Letters, vol. 21, no. 7, pp. 748-753, 2008.

[6] Y. Hu and X. Yang, "A method for obtaining first integrals and integrating factors of autonomous systems and application to Euler-Poisson equations," Reports on Mathematical Physics, vol. 58, no. 1, pp. 41-50, 2006.

[7] P. G. L. Leach and S. Bouquet, "Symmetries and integrating factors," Journal of Nonlinear Mathematical Physics, vol. 9, no. 2, pp. 73-91, 2002.

[8] S. Moyo and P. G. L. Leach, "Symmetry properties of autonomous integrating factors," SIGMA. Symmetry, Integrability and Geometry. Methods and Applications, vol. 1, Article ID 024, 12 pages, 2005.

[9] E. S. Cheb-Terrab and A. D. Roche, "Integrating factors for second-order ODEs," Journal of Symbolic Computation, vol. 27, no. 5, pp. 501-519, 1999.

[10] S. C. Anco and G. Bluman, "Integrating factors and first integrals for ordinary differential equations," European Journal of Applied Mathematics, vol. 9, no. 3, pp. 245-259, 1998.

[11] C. Muriel and J. L. Romero, "First integrals, integrating factors and $\lambda$-symmetries of second-order differential equations," Journal of Physics A, vol. 42, no. 36, Article ID 365207, 17 pages, 2009.

[12] C. Muriel and J. L. Romero, "Integrating factors and $\lambda$-symmetries," Journal of Nonlinear Mathematical Physics, vol. 15, no. 3, pp. 300-309, 2008.

[13] C. Muriel and J. L. Romero, " $\mathrm{C}^{\infty}$-symmetries and reduction of equations without Lie point symmetries," Journal of Lie Theory, vol. 13, no. 1, pp. 167-188, 2003.

[14] V. K. Chandrasekar, M. Senthilvelan, and M. Lakshmanan, “Unusual Liénard-type nonlinear oscillator," Physical Review E, vol. 72, no. 6, Article ID 066203, 8 pages, 2005.

[15] V. K. Chandrasekar, M. Senthilvelan, and M. Lakshmanan, "A nonlinear oscillator with unusual dynamical properties," in Proceedings of the 3rd National Conference on Nonlinear Systems and Dynamics (NCNSD '06), pp. 1-4, Allied Publishers, Chennai, India, 2006.

[16] V. K. Chandrasekar, M. Senthilvelan, and M. Lakshmanan, "On the complete integrability and linearization of certain second-order nonlinear ordinary differential equations," Proceedings of the Royal Society A, vol. 461, no. 2060, pp. 2451-2476, 2005.

[17] V. K. Chandrasekar, S. N. Pandey, M. Senthilvelan, and M. Lakshmanan, "A simple and unified approach to identify integrable nonlinear oscillators and systems," Journal of Mathematical Physics, vol. 47, no. 2, Article ID 023508, 2006.

[18] V. K. Chandrasekar, M. Senthilvelan, A. Kundu, and M. Lakshmanan, "A nonlocal connection between certain linear and nonlinear ordinary differential equations/oscillators," Journal of Physics A, vol. 39, no. 31, pp. 9743-9754, 2006.

[19] C. Muriel and J. L. Romero, "Symmetries and linerization of ordinary differential equations through nonlocal transformations," in Proceedings of the 3rd Conference on Nonlinear Science and Complexity, pp. 88-92, Ankara, Turkey, 2010. 
[20] T. J. Gordon, "On the symmetries and invariants of the harmonic oscillator," Journal of Physics A, vol. 19, no. 2, pp. 183-189, 1986.

[21] W. Sarlet, F. Cantrijn, and M. Crampin, "Pseudo-symmetries, Noether's theorem and the adjoint equation," Journal of Physics A, vol. 20, no. 6, pp. 1365-1376, 1987.

[22] F. Mimura and T. Nono, "A new conservation law for a system of second-order differential equations," Bulletin of the Kyushu Institute of Technology. Mathematics, Natural Science, no. 41, pp. 110, 1994.

[23] C. Muriel and J. L. Romero, "Second-order ordinary differential equations and first integrals of the form $A(t, x) \dot{x}+B(t, x)$," Journal of Nonlinear Mathematical Physics, vol. 16, pp. 209-222, 2009.

[24] C. Muriel and J. L. Romero, "Nonlocal transformations and linearization of second-order ordinary differential equations," Journal of Physics A, vol. 43, no. 43, Article ID 434025, 2010. 


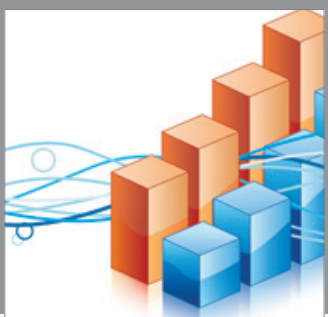

Advances in

Operations Research

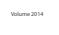

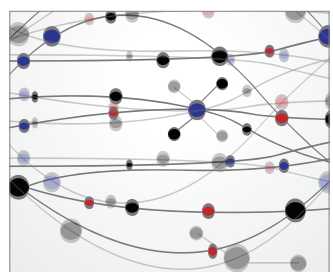

\section{The Scientific} World Journal
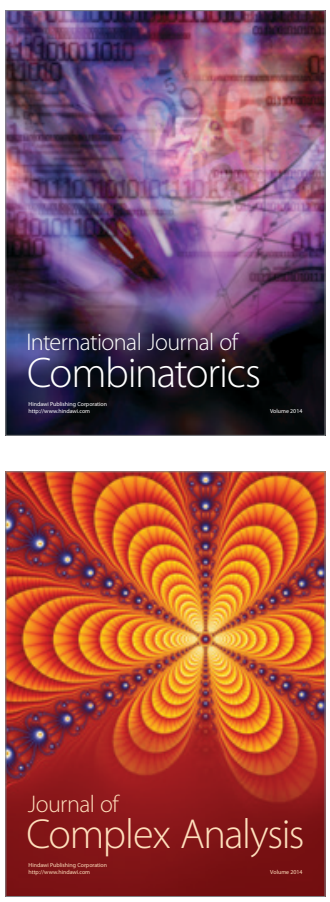

International Journal of

Mathematics and

Mathematical

Sciences
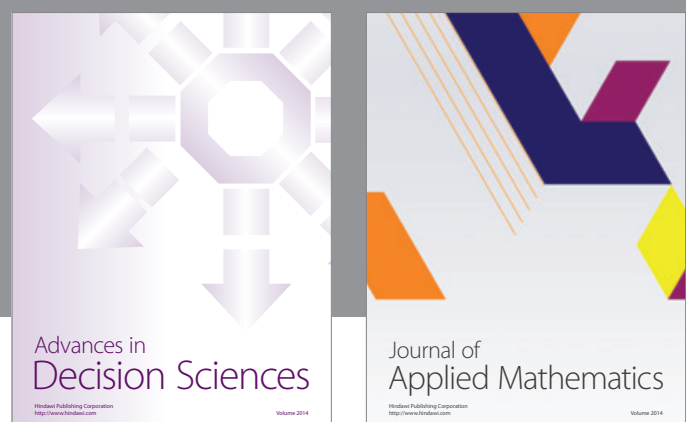

Journal of

Applied Mathematics
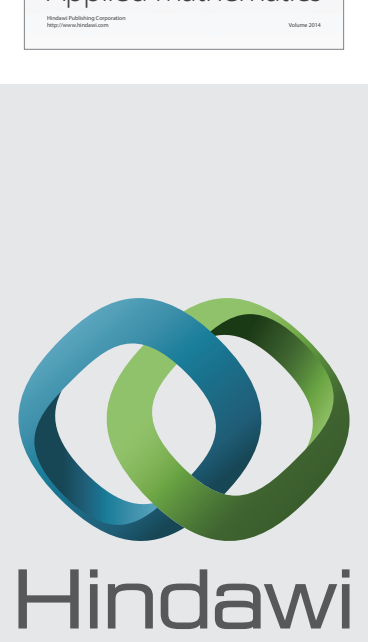

Submit your manuscripts at http://www.hindawi.com
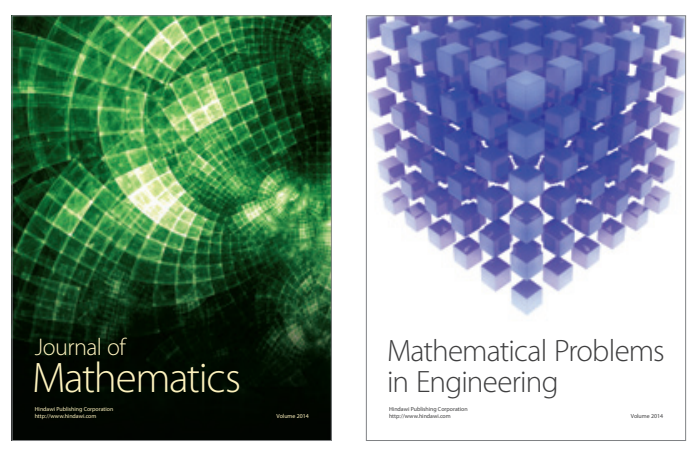

Mathematical Problems in Engineering
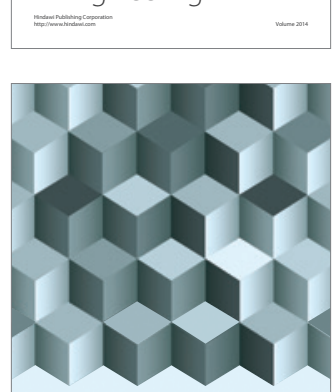

Journal of

Function Spaces
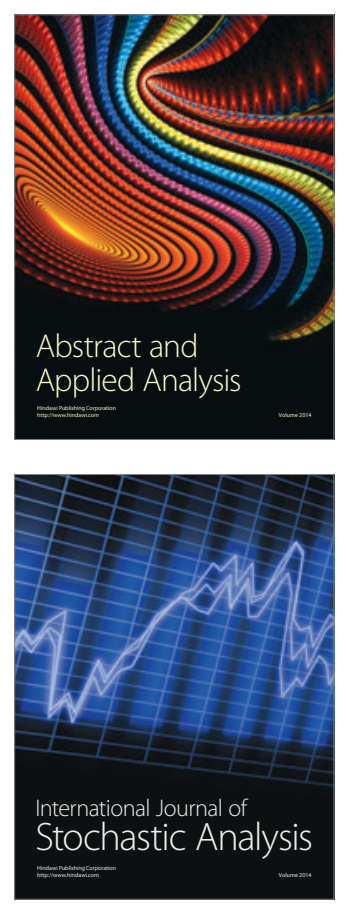

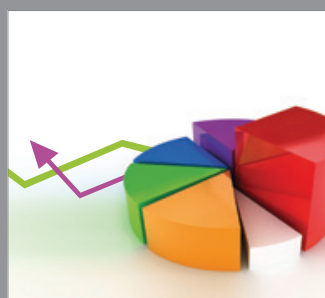

ournal of

Probability and Statistics

Promensencen
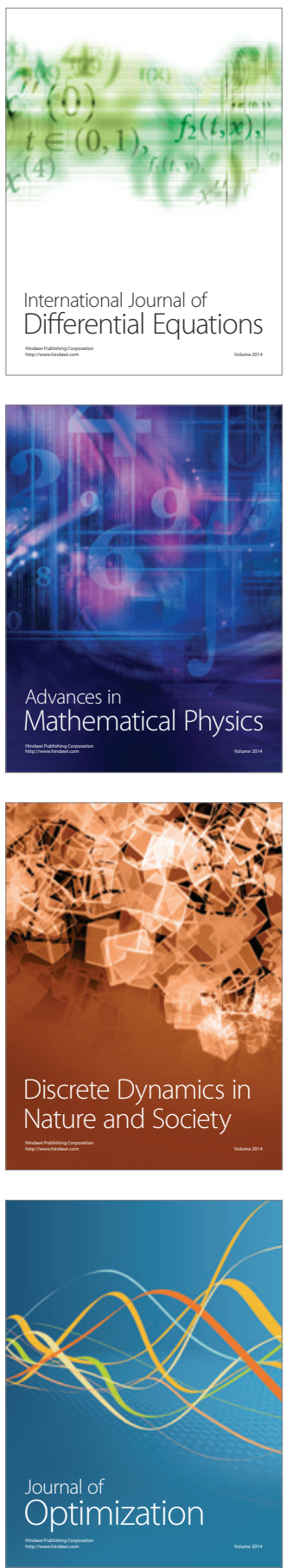